\title{
Trigonometric parallaxes of massive star forming regions: G012.88+0.48 and W33
}

\author{
K. Immer ${ }^{\star}, 1,2$, M. J. Reid ${ }^{2}$, K. M. Menten ${ }^{1}$, A. Brunthaler ${ }^{1}$, and T. M. Dame ${ }^{2}$ \\ 1 Max-Planck-Institut für Radioastronomie, Auf dem Hügel 69, 53121 Bonn, Germany \\ e-mail: kimmer@mpifr-bonn.mpg.de \\ 2 Harvard-Smithsonian Center for Astrophysics, 60 Garden Street, 02140 Cambridge, MA, USA
}

Received 26 November 2012 / Accepted 25 March 2013

\section{ABSTRACT}

\begin{abstract}
We report trigonometric parallaxes for water masers in the G012.88+0.48 region and in the massive star forming complex W33 (containing G012.68-0.18, G012.81-0.19, G012.90-0.24, G012.90-0.26), from the Bar and Spiral Structure Legacy (BeSSeL) survey using the Very Long Baseline Array. The parallax distances to all these masers are consistent with $2.40_{-0.15}^{+0.17} \mathrm{kpc}$, which locates the W33 complex and G012.88+0.48 in the Scutum spiral arm. Our results show that W33 is a single star forming complex at about two-thirds the kinematic distance of $3.7 \mathrm{kpc}$. The luminosity and mass of this region, based on the kinematic distance, have therefore been overestimated by more than a factor of two. The spectral types in the star cluster in W33 Main have to be changed by 1.5 points to later types.
\end{abstract}

Key words. astrometry - masers - parallaxes - proper motions - stars: distances - stars: formation

\section{Introduction}

W33 is a massive and very luminous star forming complex that contains typical star forming regions from quiescent infrared dark clouds to highly active, infrared bright clouds, associated with HII regions. Observations of the dust emission in the W33 complex from the APEX Telescope Large Area Survey of the GALaxy (ATLASGAL, Schuller et al. 2009) at $870 \mu \mathrm{m}$ show three large molecular clouds and several smaller dust clumps (Fig. 1). Each of the large clouds contains water masers at G012.68-0.18 (W33 B), G012.81-0.19 (W33 Main), G012.90-0.24 (W33 A), and G012.90-0.26 (W33 A) (Genzel \& Downes 1977; Jaffe et al. 1981).

To derive fundamental physical parameters of the molecular clouds such as their size, luminosity, mass, and the spectral types of embedded stars, we have to know their distances. One commonly used method is to determine the kinematic distance of a cloud from radial velocity measurements. Comparing the radial velocity of the cloud with a rotation curve of the Galaxy (e.g. Burton \& Gordon 1978; Reid et al. 2009b) yields the Galactocentric radius from which the distance of the cloud can be determined. However, in the first and fourth quadrants there are two possible distances corresponding to a given radius, complicating the distance determination. In addition, local velocity deviations due to shocks, outflows or noncircular motions can lead to large errors of the kinematic distance (Xu et al. 2006).

Kinematic distance determination for the W33 complex is complicated by a peculiar kinematic structure that was discovered via $\mathrm{H}_{2} \mathrm{CO}$ absorption line and radio recombination line observations (Bieging et al. 1978; Goss et al. 1978; Bieging et al. 1982). Two radial velocity components at 36 and $58 \mathrm{~km} \mathrm{~s}^{-1}$ were

\footnotetext{
* Member of the International Max Planck Research School (IMPRS) for Astronomy and Astrophysics at the Universities of Bonn and Cologne.
}

detected over extended parts of the region. The first component is concentrated in the northeastern part (W33 A) and the second component peaks in the southwestern part (W33 B). This velocity structure can be explained by either one connected star forming region with large internal motions at a near kinematic distance of $3.7 \mathrm{kpc}$ (corresponding to the radial velocity of $36 \mathrm{~km} \mathrm{~s}^{-1}$ ), or a superposition of independent star forming regions along the line of sight (Haschick \& Ho 1983; Goss et al. 1978; Gardner et al. 1975; Bieging et al. 1978).

Assuming a near kinematic distance of $3.7 \mathrm{kpc}$ to W33, the angular size of the complex of $15^{\prime}$ can be converted into a physical size of $16 \mathrm{pc}$. Stier et al. (1984) determined a total infrared luminosity of $\sim 2 \times 10^{6} L_{\odot}$ for W33 at this distance. Using ${ }^{13} \mathrm{CO}$ maps and a typical value of $n\left({ }^{13} \mathrm{CO}\right) / n\left(\mathrm{H}_{2}\right)=4 \times$ $10^{-7}$, Goldsmith \& Mao (1983) estimated the total mass of the complex to be between 0.2 and $2 \times 10^{6} M_{\odot}$. From radio continuum observations, Haschick \& Ho (1983) inferred the existence of an OB star cluster in W33 Main with individual spectral types ranging from $\mathrm{O} 6$ to $\mathrm{B} 0$, based on a kinematic distance of $4 \mathrm{kpc}$.

W33 A is a well-studied star forming region. The source has a high far-infrared luminosity of $10^{5} L_{\odot}$ (Faúndez et al. 2004 ) at the previously assumed kinematic distance of $3.7 \mathrm{kpc}$. Galván-Madrid et al. (2010) detected parsec-scale filaments of cold molecular gas around two dust cores. The interaction of these filaments might have triggered the star formation activity in the cores. The brightest core drives a strong outflow and the dynamics in this core show evidence for a rotating disk, perpendicular to the outflow. These results are supported by $\mathrm{CO}$ observations of Davies et al. (2010) which also suggest the presence of a rotationally-flattened cool molecular envelope. Br $\gamma$ emission in W33 A seems to trace a fast bipolar wind which has the same orientation as the large-scale outflow (Davies et al. 2010). The colder and more massive second core in W33 A seems to be 


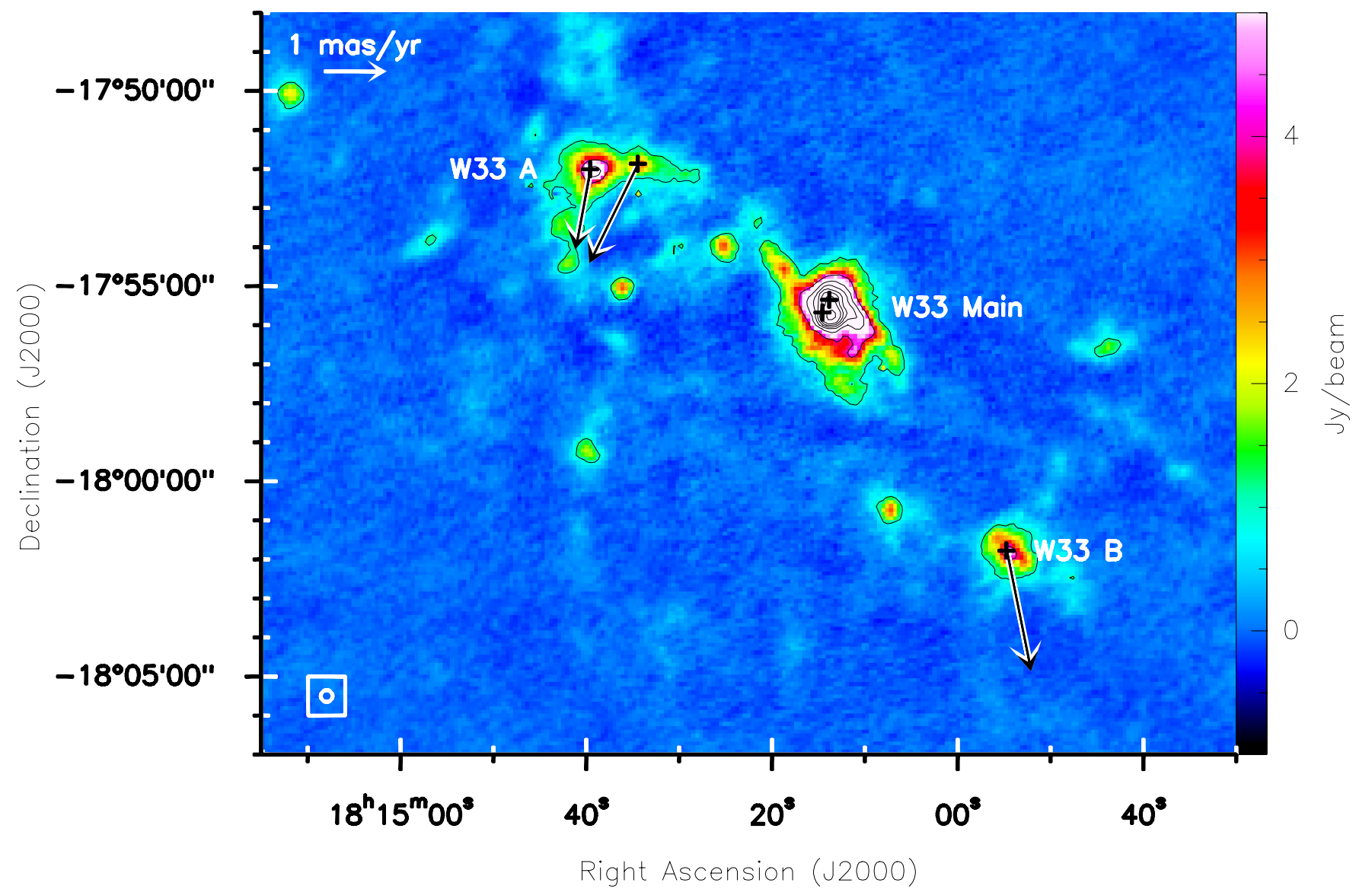

Fig. 1. $870 \mu \mathrm{m}$ dust emission of the W33 complex from the ATLASGAL survey with contour levels of 1, 4, 7, 10, 13, 16, 20, and 30 Jy beam ${ }^{-1}$. The positions of the water masers are indicated with black crosses. The arrows show the internal motion vectors of the two clouds W33 A and W33 B in the reference frame of W33 Main. The length of the arrow in the upper left corner corresponds to a motion vector of $1 \mathrm{mas} \mathrm{yr}^{-1}$. The circle in the lower left corner shows the beam of the ATLASGAL observations. G012.88+0.48 is located at an angular distance of about 0.7 degrees to the north of W33, outside of this figure.

in an earlier evolutionary stage, driving a more modest outflow (Galván-Madrid et al. 2010).

G012.88+0.48 is a high-mass protostellar object (Sridharan et al. 2002; Beuther et al. 2002) which is prominent for its strong maser emission from the $\mathrm{OH}, \mathrm{H}_{2} \mathrm{O}$, and $\mathrm{CH}_{3} \mathrm{OH}$ molecules. $\mathrm{G} 012.88+0.48$ has a radial velocity of $33.8 \mathrm{~km} \mathrm{~s}^{-1}$ (Sridharan et al. 2002), locating the star forming region at a distance of $3.6 \mathrm{kpc}$, assuming it is at its near-kinematic distance. This soure is projected about 0.7 degrees from the main star forming regions of W33. Sridharan et al. (2002) and Beuther et al. (2002) determined the luminosity and mass of this star forming region to be $L \sim 3 \times 10^{5} L_{\odot}$ and $M \sim 3 \times 10^{3} M_{\odot}$ at this distance. $\mathrm{Xu}$ et al. (2011) determined the trigonometric parallax for the $12.2 \mathrm{GHz}$ methanol masers in this source. They obtained a value of $0.428 \pm 0.022$ mas which corresponds to a distance of $2.34 \mathrm{kpc}$, revising the kinematic distance by a factor of 0.65 $(=2.34 \mathrm{kpc} / 3.6 \mathrm{kpc})$. If $\mathrm{G} 012.88+0.48$ is associated with the W33 complex, then the kinematic distances of the complex are too large. Alternatively, it is possible that G012.88+0.48 is not associated with W33 and is substantially closer to the Sun.

Other recent trigonometric parallax measurements have shown that kinematic distance measurements can be wrong by more than a factor of two (e.g. Reid et al. 2009b; Sato et al. 2010a). Trigonometric parallax observations of maser sources, however, have proven to be a reliable method to accurately measure distances to star forming regions whose accuracy is limited only by observational uncertainties.
The aim of this project is to obtain distances to the water masers in the W33 complex from trigonometric parallax observations and, therefore, address the question of whether the molecular clouds in W33 belong spatially to one connected star forming complex or are at different distances and projected near each other on the sky. Also, solid distances will provide the basis for more accurate values for the luminosity and mass of the complex.

The observations presented in this paper are part of the Bar and Spiral Structure Legacy (BeSSeL) Survey ${ }^{1}$ (Brunthaler et al. 2011), a National Radio Astronomy Observatory's ${ }^{2}$ Very Long Baseline Array (VLBA) key project which will determine the distances to hundreds of star forming regions. We report results of the parallax and proper motion measurements toward the water masers in the sources G012.88+0.48 and the massive star forming complex W33.

\section{Observation and data reduction}

The massive star forming region $\mathrm{G} 012.88+0.48$ and the W33 complex (G012.68-0.18, G012.81-0.19, G012.90-0.24, G012.90-0.26) were observed in the $6_{16}-5_{23}$ transition of the

\footnotetext{
http://bessel.vlbi-astrometry.org/index.shtml

2 The National Radio Astronomy Observatory (NRAO) is a facility of the National Science Foundation operated under cooperative agreement by Associated Universities, Inc.
} 
K. Immer et al.: Trigonometric parallaxes of G012.88+0.48 and W33

Table 1. Coordinates, line-of-sight velocities, and peak flux densities of the background quasars and the strongest maser spots.

\begin{tabular}{|c|c|c|c|c|c|c|}
\hline Source & $\begin{array}{c}\text { RA (J2000) } \\
{[\text { hh mm ss.ssss] }}\end{array}$ & $\begin{array}{c}\operatorname{Dec}(\mathrm{J} 2000) \\
{\left[\mathrm{dd}{ }^{\prime \prime}, " . .\right.} \\
\end{array}$ & $\begin{array}{c}V_{\mathrm{LSR}} \\
{\left[\mathrm{km} \mathrm{s}^{-1}\right]}\end{array}$ & $\begin{array}{c}F_{\text {Peak }} \\
{\left[\text { Jy beam }^{-1} \text { ] }\right.}\end{array}$ & $\begin{array}{c}\mathrm{rms} \\
{\left[\mathrm{Jy} \mathrm{beam}^{-1} \text { ] }\right.}\end{array}$ & Cloud association \\
\hline $\mathrm{J} 1808-1822$ & 180855.5150 & -182253.383 & & 0.012 & 0.001 & \\
\hline $\mathrm{J} 1809-1520$ & 180910.2094 & -152009.699 & & 0.022 & 0.001 & \\
\hline J1825-1718 & 182536.5323 & -171849.848 & & 0.096 & 0.001 & \\
\hline G012.68-0.18 & 181354.7457 & -180146.588 & 59.3 & 132.5 & 0.001 & W33 B \\
\hline G012.81-0.19 & 181413.8283 & -175521.035 & $34.1 /-1.4$ & $1.0 / 0.2$ & $0.018 / 0.007$ & W33 Main \\
\hline G012.90-0.24 & 181434.4366 & -175151.891 & 34.9 & 18.3 & 0.081 & W33 A \\
\hline G012.90-0.26 & 181439.5714 & -175200.382 & 37.0 & 3.0 & 0.016 & W33 A \\
\hline $\mathrm{G} 012.88+0.48$ & 181151.4939 & -173128.911 & 29.4 & 23.3 & 0.133 & \\
\hline
\end{tabular}

Notes. Columns 2, 3, and 4 list the coordinates and line-of-sight velocities of the background quasars and the maser spots that were used for parallax fitting. The peak flux densities of the background quasars and the strongest maser spots and the rms of the reference channel images, obtained from epoch 2011 May 21, are listed in Cols. 5 and 6. The last column shows with which cloud the masers in the W33 complex are associated.

$\mathrm{H}_{2} \mathrm{O}$ molecule (rest frequency $22.23508 \mathrm{GHz}$ ), using the VLBA. The general observation setup and data calibration procedures are described in Reid et al. (2009a). Here, we only describe the details specific to the present sources.

All water masers were observed at nine epochs (VLBA program BR145I) with a total observing time per epoch of $\sim 7 \mathrm{~h}$. The observing dates (2010 Sep. 19, 2010 Oct. 22, 2010 Dec. 19, 2011 Feb. 15, 2011 Mar. 26, 2011 May 21, 2011 Jul. 30, 2011 Sep. 20, 2012 Jan. 03) were selected to well sample the Right Ascension parallax signature in time, since the Declination parallax signature is much smaller. However, due to bad weather conditions, the data of the first epoch could not be used for the parallax measurements.

Three different quasars, J1808-1822, J1809-1520, and J1825-1718, from the VLBA calibrator search for the BeSSeL survey (Immer et al. 2011) and the ICRF2 catalog (Fey et al. 2009), were observed for background position references. The background source J1809-1520 could only be detected in the first four epochs, and thus was not used for the parallax determination. Four adjacent frequency bands with $4 \mathrm{MHz}$ bandwidth each were used in right and left circular polarization. The maser signals were centered in the second band at an LSR velocity, $V_{\mathrm{LSR}}$, of $45 \mathrm{~km} \mathrm{~s}^{-1}$ for all masers. The channel spacing of the observations is $0.42 \mathrm{~km} \mathrm{~s}^{-1}$.

In order to correct for instrumental phase offsets between the frequency bands, observations of the calibrator J1800+3848 from the ICRF2 catalog were used. The strongest maser feature in the water maser G012.68-0.18 at a $V_{\mathrm{LSR}}$ of $59.3 \mathrm{~km} \mathrm{~s}^{-1}$ was used for the phase-referencing of the data.

The water masers G012.81-0.19, G012.90-0.24, G012.90-0.26, and G012.88+0.48 were much weaker than G012.68-0.18 and were calibrated using G012.68-0.18 as phase reference. This yields positions relative to G012.68-0.18, from which relative parallaxes can be estimated.

For the data reduction, we used the software Parseltongue ${ }^{3}$ (Kettenis et al. 2006), a scripting interface for NRAO's Astronomical Image Processing System (AIPS). After calibrating the data, the maser emission and the continuum sources were imaged with the AIPS task IMAGR with a circular Clean beam of 2 mas for all epochs. The positions of the masers and the background sources J1808-1822 and J1825-1718 were then determined by fitting Gaussian brightness distributions to the images (see Reid et al. 2009a). Absolute positions of the strongest maser spot in G12.68-0.81 were derived relative to the two

\footnotetext{
3 http://www . radionet-eu.org/rnwiki/ParselTongue
}

background sources. For the remaining water masers, relative positions to G012.68-0.18 were determined. To obtain absolute parallaxes, we added the positions of G012.68-0.18 relative to both background quasars to the position measurements of these water masers.

The positions were modeled with a sinusoidal parallax signature and linear proper motions in each coordinate. Since systematic errors (typically from unmodeled atmospheric delays) are normally much larger than random noise, the formal position errors from the Gaussian fits are mostly too small to account for the total error of the observations which leads to high $\chi^{2}$ values in the modeling process. The magnitude of the systematic errors can only be inferred from the quality of the parallax fit. Thus, we defined "error-floors" for the Right Ascension and Declination maser positions and added those in quadrature to the Gaussian fit errors. The "error-floor" values were adjusted until the $\chi^{2}$ value per degree of freedom was close to unity for both coordinates (see Reid et al. 2009a).

In order to determine the internal gas kinematics in each source, we measured the proper motions for all maser spots that were detected in at least three epochs without solving for the parallax parameter.

\section{Results}

Table 1 gives the coordinates (Cols. 2, 3), line-of-sight velocities (Col. 4), and peak flux densities from epoch 2011 May 21 (Col. 5) of the background quasars and the maser spots that were used for the parallax fits. Column 6 lists the rms brightness of the reference channel images, obtained from epoch 2011 May 21. In the last column, we show the associations of the masers in the W33 complex.

The parallax and proper motion results of all masers are summarized in Table 2. Columns 2-4 give the line-of-sight velocities of the masing and thermal gas and the parallaxes of the maser sources. In the sixth and seventh columns, the proper motions in eastward and northward directions are listed.

One goal of our observations is to estimate the distance and proper motion of the central object in each source. In G012.68-0.18, we obtained the proper motion of the central object by fitting a model for expanding outflows (Sato et al. 2010b) to the proper motions of all maser spots. In the other sources, the small number of maser spots and their distribution did not allow the fitting of this model to their proper motions. Instead, the presented values are averages of the proper motions of all maser spots in each source. 
Table 2. Parallax and proper motion results.

\begin{tabular}{lcccccc}
\hline \multicolumn{1}{c}{$\begin{array}{c}\text { Maser } \\
\text { Source }\end{array}$} & $\begin{array}{c}V_{\text {LSR,maser }} \\
\mathrm{km} \mathrm{s}^{-1}\end{array}$ & $\begin{array}{c}V_{\text {LSR,thermal }}{ }^{a} \\
\mathrm{~km} \mathrm{~s}^{-1}\end{array}$ & $\begin{array}{c}\text { Parallax } \pi \\
{[\mathrm{mas}]}\end{array}$ & $\begin{array}{c}\text { Distance } d \\
{[\mathrm{kpc}]}\end{array}$ & $\begin{array}{c}\mu_{x} \\
{\left[\mathrm{mas} \mathrm{yr}^{-1}\right]}\end{array}$ & $\begin{array}{c}\mu_{y} \\
{\left[\mathrm{mas} \mathrm{yr}^{-1}\right]}\end{array}$ \\
\hline G012.68-0.18 (W33 B) & 59.3 & $\sim 55$ & $0.416 \pm 0.028$ & $2.40_{-0.15}^{+0.17}$ & $-1.00 \pm 0.30$ & $-2.85 \pm 0.29$ \\
G012.81-0.19 (W33 Main) & -1.4 & $\sim 36$ & $0.343 \pm 0.037$ & $2.92_{-0.28}^{+0.35}$ & $-0.24 \pm 0.17$ & $+0.54 \pm 0.12$ \\
G012.81-0.19 (W33 Main) & 34.1 & $\sim 36$ & $0.343 \pm 0.037$ & $2.92_{-0.28}^{+0.35}$ & $-0.60 \pm 0.11$ & $-0.99 \pm 0.13$ \\
G012.90-0.24 (W33 A) & 34.9 & $\sim 36$ & $0.408 \pm 0.025$ & $2.45_{-0.14}^{+0.16}$ & $+0.19 \pm 0.08$ & $-2.52 \pm 0.32$ \\
G012.90-0.26 (W33 A) & 37.0 & $\sim 37$ & $0.396 \pm 0.032$ & $2.53_{-0.19}^{+0.22}$ & $-0.36 \pm 0.08$ & $-2.22 \pm 0.13$ \\
\hline G012.88+0.48 & 29.4 & $\sim 34$ & $0.340 \pm 0.036$ & $2.94_{-0.28}^{+0.35}$ & $+0.12 \pm 0.13$ & $-2.66 \pm 0.23$ \\
\hline
\end{tabular}

Notes. ${ }^{(a)}$ Sridharan et al. (2002); Purcell et al. (2012); Wienen et al. (2012). Columns 6 and 7 give the proper motions in eastward and northward directions. The proper motions of the first maser are obtained from fitting a model of expanding outflows to all maser spots. The proper motions of the remaining sources are averages of the proper motions of all maser spots in each source.

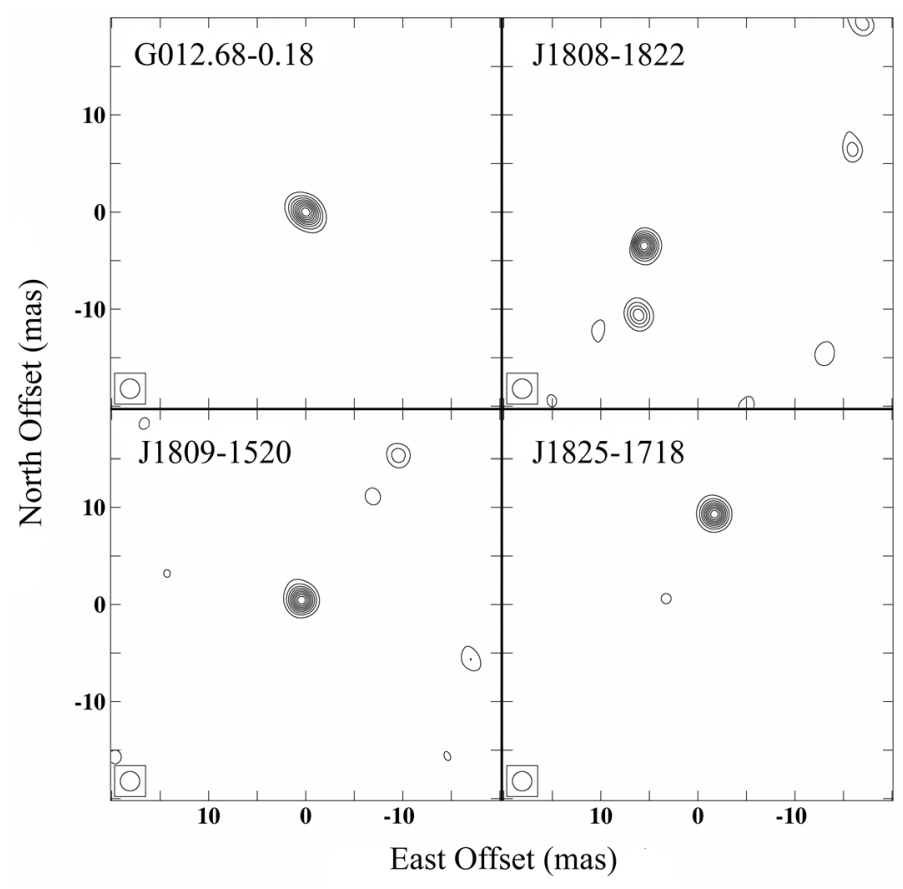

Fig. 2. Left upper panel: maser emission in reference channel $\left(59.3 \mathrm{~km} \mathrm{~s}^{-1}\right)$. Right upper, left and right lower panel: compact emission of the three background quasars J1808-1822, J1809-1520, and J1825-1718, which were phase-referenced to the reference maser channel. The images are obtained from epoch 2011 May 21. The contour levels are 10 to $100 \%$ in steps of $10 \%$ of the maximum in each panel (G012.68-0.18: 132.5 Jy beam ${ }^{-1}$, J1808-1822: $12.3 \mathrm{mJy} \mathrm{beam}^{-1}$, J1809-1520: $22.1 \mathrm{mJy}_{\text {beam }}{ }^{-1}$, J1825-1718: $96.1 \mathrm{mJy} \mathrm{beam}^{-1}$ ). The restoring beam is shown in the lower left corner.

\subsection{W33}

Figure 1 shows the dust emission of the W33 complex from the ATLASGAL survey at $870 \mu \mathrm{m}$. The three most massive clouds W33 A, W33 Main, and W33 B are indicated. The water masers are marked with black crosses.

Water maser spots were detected in an $0{ }^{\prime} 15 \times 0.15$ area in W33 B (G012.68-0.18), covering a velocity range of $57-63 \mathrm{~km} \mathrm{~s}^{-1}$. Figure 2 shows that the maser emission in the reference channel $\left(V_{\mathrm{LSR}}=59.3 \mathrm{~km} \mathrm{~s}^{-1}\right.$, left panel) as well as the emission of the three background quasars J1808-1822 (right upper panel), J1809-1520 (left lower panel), and J1825-1718 (right lower panel) are dominated by one compact component.
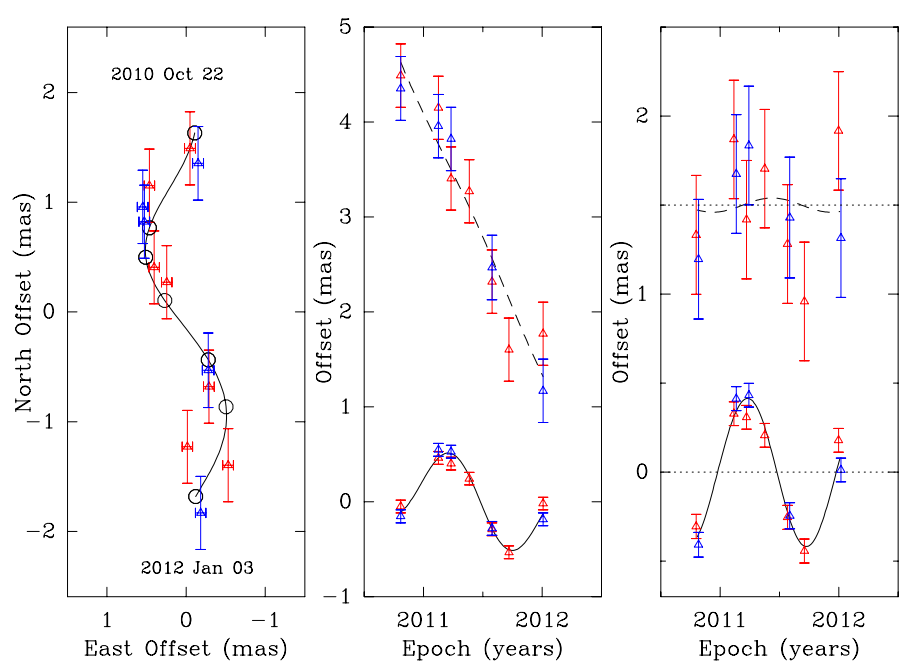

Fig. 3. Parallax and proper motion fits of G012.68-0.18, yielding a parallax of $(0.416 \pm 0.028)$ mas. The positions of the reference maser spot at 59.3 (triangles) $\mathrm{km} \mathrm{s}^{-1}$ were measured relative to the background quasars J1808-1822 (blue) and J1825-1718 (red). Left panel: position on the sky with a label for the first and last epoch. Middle panel: east (continuous line) and North (dashed line) position offsets with parallax and proper motion fits versus time. Right panel: right ascension (continuous line) and Declination (dashed line) parallax fits with the best-fit proper motions removed, showing only the parallax signature.

Since the quasar J1809-1520 was detected only in the first four epochs, we determined the position of the reference maser spot only relative to the background quasars J1808-1822 and J1825-1718 as a function of time (see Fig. 3). For three epochs (2010 Oct. 22, 2010 Dec. 19, 2011 Sep 20), we used a different maser spot at the same velocity as position reference since the original reference maser spot, used in the other epochs, was too weak to serve as a phase reference.

The positions of the reference maser spot in G012.68-0.18 relative to background quasars were fitted with the sinusoidal parallax signature and a linear proper motion. We obtained parallax estimates of $\pi=0.462 \pm 0.034$ mas and $\pi=0.387 \pm$ 0.037 mas, relative to the background quasars J1808-1822 and J1825-1718, respectively. Since both results are consistent within their joint uncertainty, we combined the two data sets and repeated the fitting, yielding a value of $\pi=0.416 \pm 0.028$ mas which corresponds to a distance of $2.40_{-0.15}^{+0.17} \mathrm{kpc}$ (see Fig. 3).

We fitted the proper motions of all maser spots in G012.68-0.18, detected in at least three epochs, with a model 


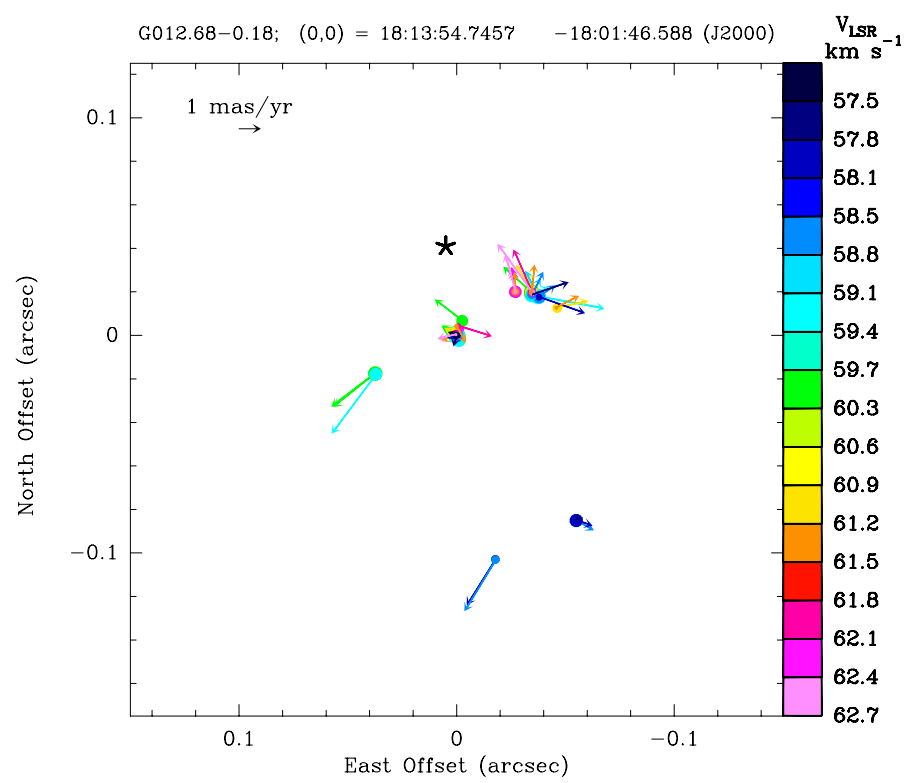

Fig. 4. Positions of all maser spots in G012.68-0.18 that have been detected in at least three epochs (positions from epoch 2011 May 21). The size of the spots scales logarithmically with the flux density of the spots. The asterisk marks the position of the central star which has an error of $\sim 0.1^{\prime \prime}$ in both coordinates. The arrows show the proper motions of the maser spots in the reference frame of the central star.
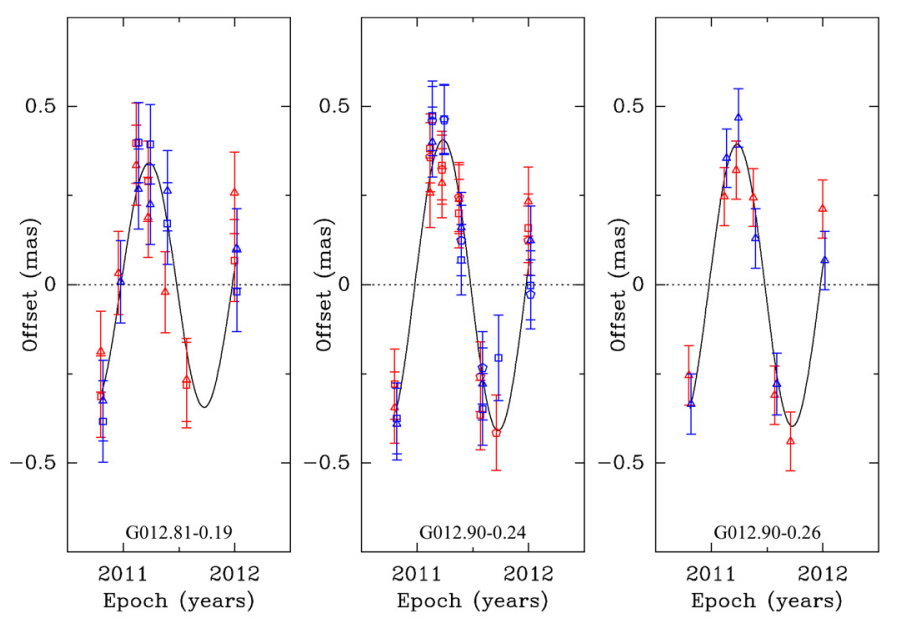

Fig. 5. Right ascension parallax fits of G012.81-0.19 (left), G012.90-0.24 (middle), and G012.90-0.26 (right), yielding absolute parallaxes of $0.343 \pm 0.037$ mas, $0.408 \pm 0.025$ mas, and $0.396 \pm 0.032$ mas, respectively. The positions of the maser spots were measured relative to the water maser G012.68-0.18 and consecutively to the two background quasars J1808-1822 (blue) and J1825-1718 (red). Left panel: positions of the maser spots at 34.1 (triangles) and -1.4 (squares) $\mathrm{km} \mathrm{s}^{-1}$. Middle panel: positions of the maser spots at 34.5 (triangles), 34.9 (squares), and 35.3 (pentagons) $\mathrm{km} \mathrm{s}^{-1}$. Right panel: positions of the maser spot at 37.0 (triangles) $\mathrm{km} \mathrm{s}^{-1}$.

for expanding outflows with the position and proper motion of the central driving source as free parameters (for more details about the model see Sato et al. 2010b). The proper motion of the central star is $\mu_{x}=-1.00 \pm 0.30 \mathrm{mas} \mathrm{yr}^{-1}$ and $\mu_{y}=-2.85 \pm 0.29 \mathrm{mas} \mathrm{yr}^{-1}$. Figure 4 shows the positions of the maser spots with their proper motions in the reference frame of the central source. Observations with the Submillimeter Array (SMA) at $230 \mathrm{GHz}$ (Immer et al., in prep.) show that the reference maser spot is located in a dust core, offset by $\sim 0.1^{\prime \prime}$ from

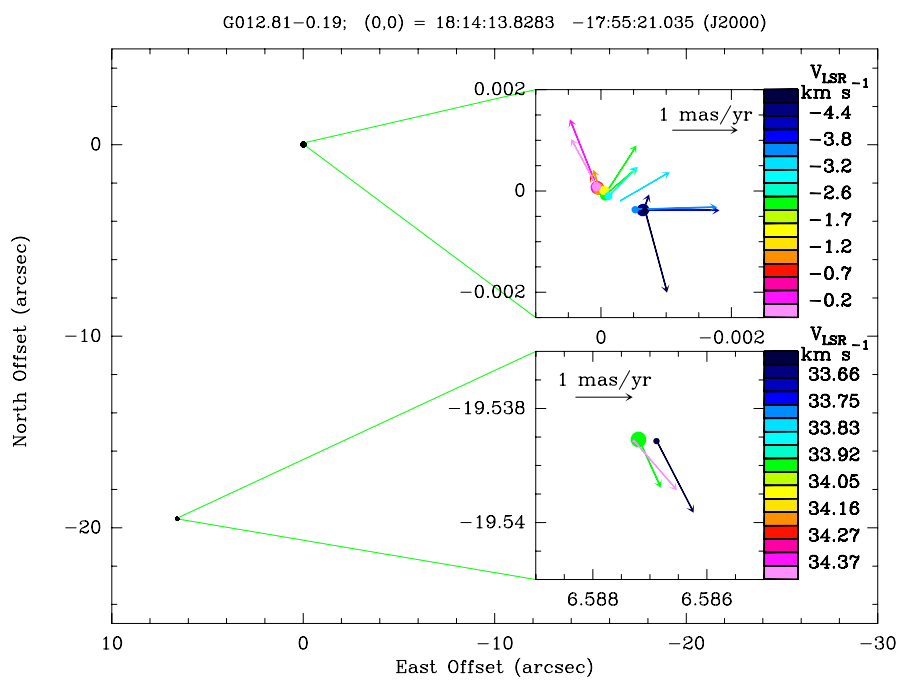

Fig. 6. Left panel: positions of all maser spots in G012.81-0.19 that have been detected in at least three epochs (positions from epoch 2011 May 21). Inside panels: zoom-in on the two maser spot groups at $34 \mathrm{~km} \mathrm{~s}^{-1}$ (lower panel) and $-1 \mathrm{~km} \mathrm{~s}^{-1}$ (upper panel). The maser spots in the upper panel are ordered along a ridge. The size of the spots scales logarithmically with the flux density of the spots. The arrows show the absolute proper motions of the maser spots.

the submillimeter continuum peak. The radial velocity of the reference maser spot is similar to the systemic velocity of the thermal gas in this cloud (55 $\mathrm{km} \mathrm{s}^{-1}$, Wienen et al. 2012).

The remaining W33 masers and G012.88+0.48 were phasereferenced to G12.68-0.18 and the positions of their strongest maser spots were determined relative to this maser, yielding relative parallaxes. To obtain absolute parallax values, the positions of $\mathrm{G} 012.68-0.18$ relative to the two background quasars were added to the position measurements of the other water masers.

In G012.81-0.19, we detected two spatially and kinematically distinct maser groups, covering velocity ranges of 33 to $35 \mathrm{~km} \mathrm{~s}^{-1}$ and -5 to $0 \mathrm{~km} \mathrm{~s}^{-1}$, separated by $\sim 21^{\prime \prime}$. The positions of two maser spots at -1.4 and $34.1 \mathrm{~km} \mathrm{~s}^{-1}$ were determined relative to G012.68-0.18. Combining the two position measurements, we obtained an absolute parallax of $\pi=0.343 \pm$ 0.037 mas (see Fig. 5, left panel). The positions of the two maser groups are shown in Fig. 6. The images on the right-hand side show both maser groups on a smaller scale with their proper motions. Averaging the proper motions of all maser spots in each group, yields a proper motion value for each velocity group (uncorrected for expansion): $\mu_{x, 34 \mathrm{~km} \mathrm{~s}^{-1}}=-0.24 \pm 0.17 \mathrm{mas} \mathrm{yr}^{-1}$ and $\mu_{y, 34 \mathrm{~km} \mathrm{~s}^{-1}}=0.54 \pm 0.12 \mathrm{mas} \mathrm{yr}^{-1}$ as well as $\mu_{x,-1 \mathrm{~km} \mathrm{~s}^{-1}}=$ $-0.60 \pm 0.11 \mathrm{mas} \mathrm{yr}^{-1}$ and $\mu_{y,-1 \mathrm{~km} \mathrm{~s}^{-1}}=-0.99 \pm 0.13 \mathrm{mas} \mathrm{yr}^{-1}$. The maser spots in the velocity group around $-1 \mathrm{~km} \mathrm{~s}^{-1}$ are ordered along a ridge with redshifted velocities at the north-east end and blueshifted velocities at the south-west end. The maser spot group at $34 \mathrm{~km} \mathrm{~s}^{-1}$ is located in a dust core, offset by $\sim 7^{\prime \prime}$ from the main continuum emission peak at $230 \mathrm{GHz}$ (Immer et al., in prep.). The radial velocity of the reference maser spot is similar to the systemic velocity of the thermal gas of $36 \mathrm{~km} \mathrm{~s}^{-1}$, which was estimated from ammonia observations of the $\mathrm{H}_{2} \mathrm{O}$ southern Galactic Plane Survey (HOPS, Purcell et al. 2012). The maser spot group at $-1 \mathrm{~km} \mathrm{~s}^{-1}$ is associated with emission from a dust core at $345 \mathrm{GHz}$ (Immer et al., in prep.). However, the radial velocities of these maser spots are very different from the systemic velocity in this source and they might trace the blueshifted shock front of an outflow. 


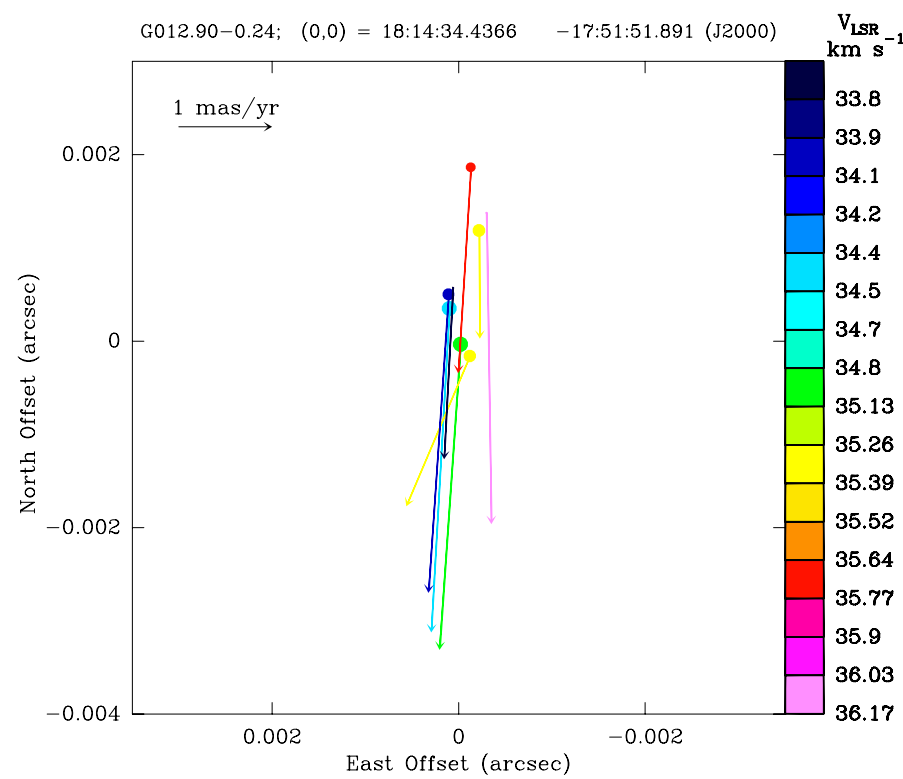

Fig. 7. Positions of all maser spots in G012.90-0.24 that have been detected in at least three epochs (positions from epoch 2011 May 21). The size of the spots scales logarithmically with the flux density of the spots. The arrows show the absolute proper motions of the maser spots.

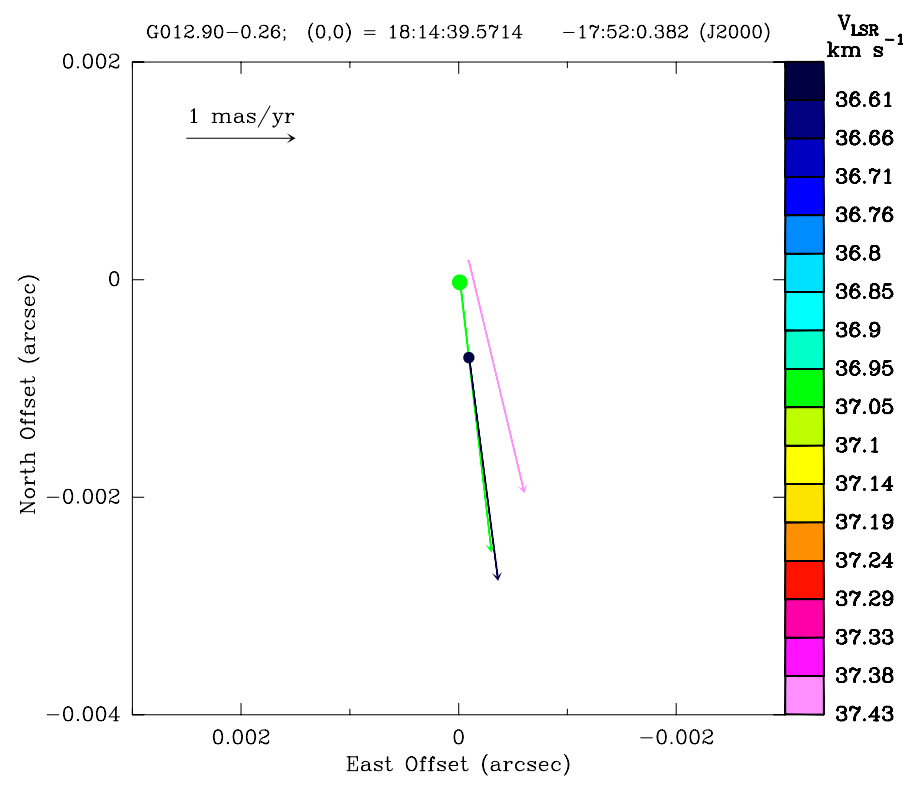

Fig. 8. Positions of all maser spots in G012.90-0.26 that have been detected in at least three epochs (positions from epoch 2011 May 21). The size of the spots scales logarithmically with the flux density of the spots. The arrows show the absolute proper motions of the maser spots.

The maser emission from G012.90-0.24 spans a velocity range of 33 to $37 \mathrm{~km} \mathrm{~s}^{-1}$. For the parallax fitting, we used the positions of three maser spots at velocities of 34.5, 34.9, and $35.3 \mathrm{~km} \mathrm{~s}^{-1}$ and obtained an absolute parallax of $\pi=0.408 \pm$ 0.025 mas (see Fig. 5, middle panel). The proper motions of the detected maser spots are very similar (Fig. 7). The proper motion of the source (uncorrected for expansion), obtained from averaging all maser spot proper motions, is $\mu_{x}=0.19 \pm 0.08$ mas yr$^{-1}$

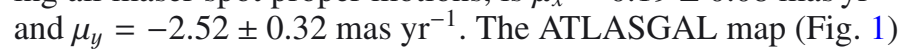
shows that the reference maser spots are offset by $\sim 8^{\prime \prime}$ from a submillimeter continuum peak. The radial velocities of the
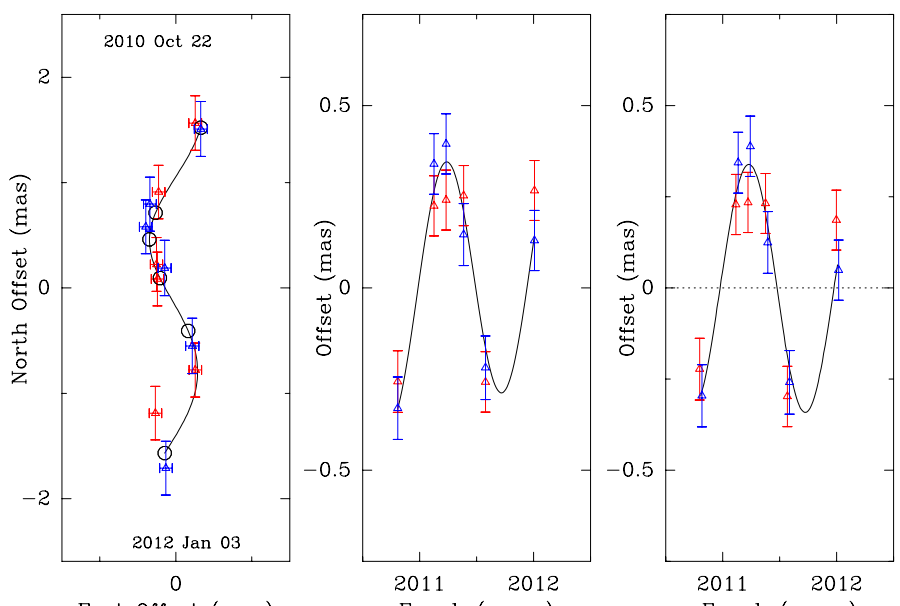

Fig. 9. Parallax and proper motion fits of G012.88+0.48, yielding an absolute parallax of $0.340 \pm 0.036$ mas. The positions of the maser spot at 29.4 (triangles) $\mathrm{km} \mathrm{s}^{-1}$ were measured relative to the water maser G012.68-0.18 and consecutively to the two background quasars J1808-1822 (blue) and J1825-1718 (red). Left panel: position on the sky with a label for the first and last epoch. Middle panel: east position offsets with parallax and proper motion fits versus time. Right panel: right ascension parallax fit with the best-fit proper motions removed, showing only the parallax signature.

reference maser spots are close to the systemic velocity of G012.90-0.24 (36 km s${ }^{-1}$, estimated from the HOPS survey).

Only three maser spots were detected in G012.90-0.26, covering a velocity range of 36 to $38 \mathrm{~km} \mathrm{~s}^{-1}$. We used the strongest maser spot at $37.0 \mathrm{~km} \mathrm{~s}^{-1}$ for the parallax fitting, yielding an absolute parallax of $\pi=0.396 \pm 0.032$ mas (see Fig. 5, right panel). Averaging the proper motions of the three maser spots, we obtain a proper motion of the source of $\mu_{x}=-0.36 \pm$ 0.08 mas yr$^{-1}$ and $\mu_{y}=-2.22 \pm 0.13$ mas yr$^{-1}$. The position of the reference maser spot is consistent with the submillimeter continuum peak MM1-SE, detected by Galván-Madrid et al. (2010) at $230 \mathrm{GHz}$. This object is associated with a faint radio source at $7 \mathrm{~mm}$ (van der Tak \& Menten 2005). The radial velocity of the reference maser spot is very close to the systemic velocity of the thermal gas in W33 A $\left(38.5 \mathrm{~km} \mathrm{~s}^{-1}\right.$, Galván-Madrid et al. 2010). A comparison of the proper motion of G012.90-0.26 with CO observations of the large-scale outflow in W33 A (Galván-Madrid et al. 2010) show that their directions do not agree. Thus, we will assume that the proper motion of this water maser is not strongly influenced by the outflow but probably reflects the motion of the central object.

\section{2. $G 012.88+0.48$}

Maser emission in the spectrum of G012.88+0.48 was detected only in the velocity range 27 to $31 \mathrm{~km} \mathrm{~s}^{-1}$. Fitting the positions of the strongest maser spot at $29.4 \mathrm{~km} \mathrm{~s}^{-1}$, we obtained an absolute parallax of $\pi=0.340 \pm 0.036$ mas (see Fig. 9), which is consistent within $2 \sigma$ (joint uncertainty) with the parallax result of $\mathrm{Xu}$ et al. (2011) for $12.2 \mathrm{GHz}$ methanol masers in this star forming region.

Figure 10 shows two groups of maser spots, separated by $\sim 0.25^{\prime \prime}$. The spatial distribution of the spots in the western group shows an arc-like morphology. The combination with the proper motions of these spots seems to indicate that the water masers trace a shock front in this source. Methanol maser observations in $\mathrm{G} 012.88+0.48$ at $6.7 \mathrm{GHz}$ with the Japanese VLBI Network 


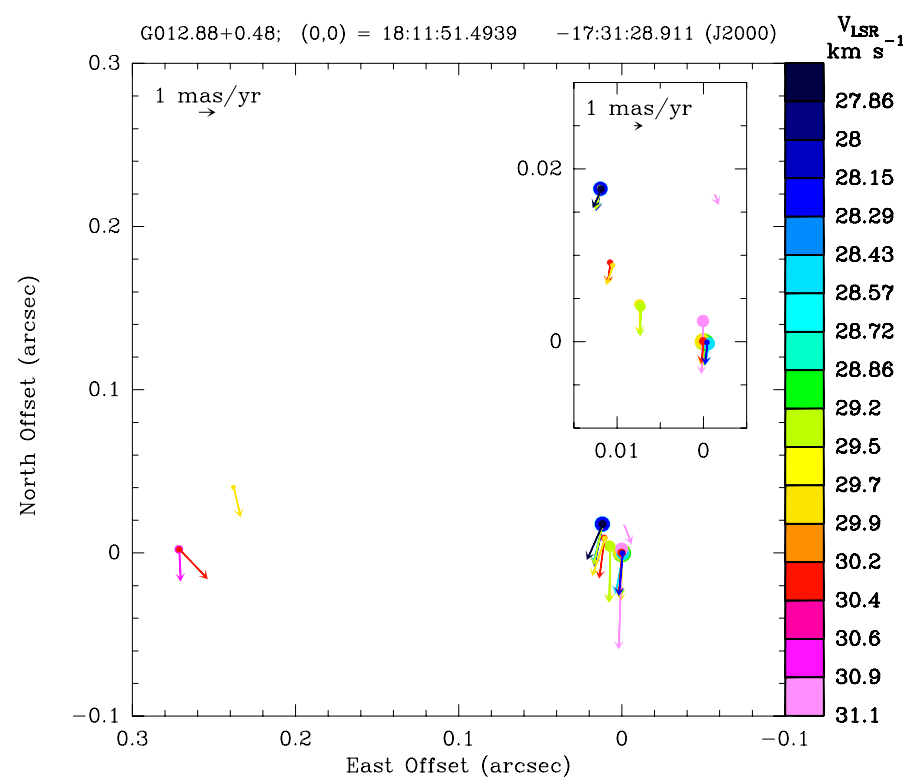

Fig. 10. Positions of all maser spots in G012.88+0.48 that have been detected in at least three epochs (positions from epoch 2011 May 21). The size of the spots scales logarithmically with the flux density of the spots. The arrows show the absolute proper motions of the maser spots. The smaller picture is a zoom on the western group of maser spots. These spots might be tracing a bow shock in this source.

and the East-Asian VLBI Network present a similar picture with a group of maser spots arranged in an arc-like structure with line-of-sight velocities from 30 to $40 \mathrm{~km} \mathrm{~s}^{-1}$ (Fujisawa et al. 2012 , in prep.). This maser group is offset from our western group by $-0.3^{\prime \prime}$ and $-1.2^{\prime \prime}$ in east-west and north-south direction, respectively.

We averaged the proper motions of all water maser spots to obtain one proper motion for G012.88+0.48, yielding $\mu_{x}=$ $0.12 \pm 0.13 \mathrm{mas} \mathrm{yr}^{-1}$ and $\mu_{y}=-2.66 \pm 0.23 \mathrm{mas} \mathrm{yr}^{-1}$.

\section{Discussion}

The absolute parallaxes of G012.81-0.19, G012.88+0.48, G012.90-0.24, and G012.90-0.26 are consistent with a parallax of 0.416 mas within $2 \sigma$, i.e. the distances to these masers are in accordance with the distance to G012.68-0.18 (2.40 kpc).

Comparing the coordinates of the W33 complex with a CO latitude-velocity map from the survey of Dame et al. (2001) (Fig. 11), we find that the complex is associated with a bright cloud at the same latitude with a mean velocity of $34.6 \mathrm{~km} \mathrm{~s}^{-1}$, which falls along the longitude-velocity locus of the Scutum spiral arm.

Since all water masers in the W33 complex are at a similar distance, we conclude that the molecular clouds W33 A, W33 Main, and W33 B are connected and belong to the same star forming complex. Our parallax distance to this star forming cluster is a factor of $0.65(=2.4 \mathrm{kpc} / 3.7 \mathrm{kpc})$ smaller than the kinematic distance. Thus, the luminosity and mass of this complex have been overestimated in the past by a factor of more than two and should be revised to $L \sim 8 \times 10^{5} L_{\odot}$ and $M \sim$ $(0.8-8) \times 10^{5} M_{\odot}$.

The spectral types of the stars in the W33 Main cluster were determined from the estimated number of Lyman continuum photons needed to ionize the material in the surrounding $\mathrm{H}$ II region. Since the number of photons depends on distance squared, the values have been overestimated by a factor of more than two, changing the spectral types by 1.5 points to later types, i.e., a star

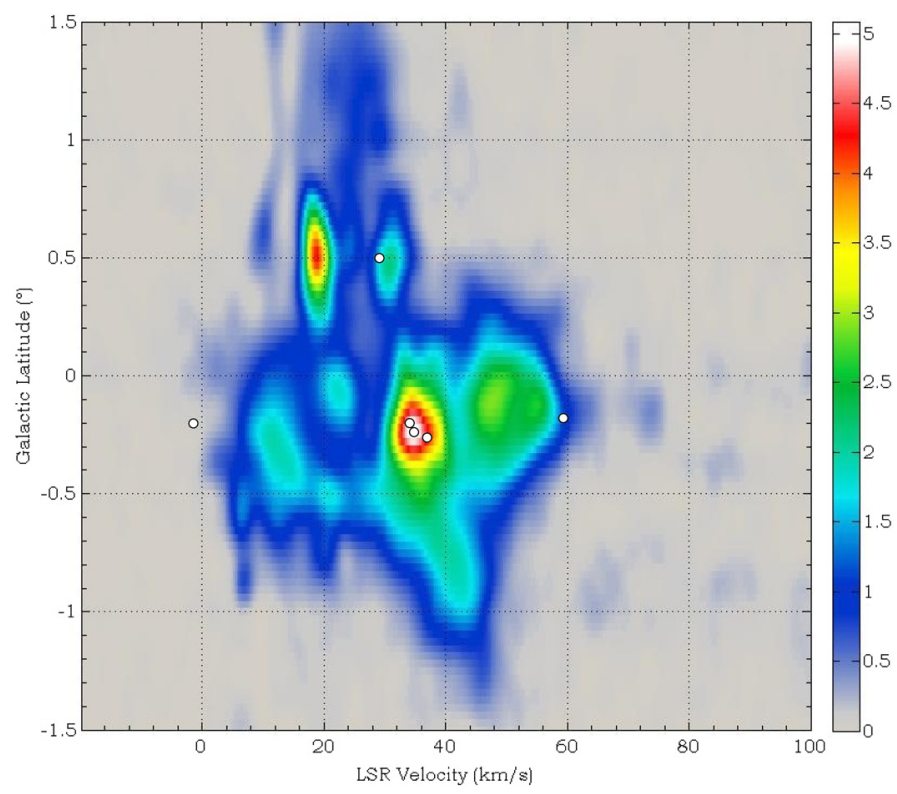

Fig. 11. Velocities of the water maser groups in the CO latitude-velocity plot (white dots). The $\mathrm{CO}$ emission was integrated over a longitude range from $12.625^{\circ}$ to $13.0^{\circ}$. The units of the color bar are degrees Kelvin. The water masers in W33 and in G012.88+0.48 are associated with clouds at $34.6 \mathrm{~km} \mathrm{~s}^{-1}$ and $30.9 \mathrm{~km} \mathrm{~s}^{-1}$, respectively. The water masers at $-1 \mathrm{~km} \mathrm{~s}^{-1}$ (W33 Main) and $58 \mathrm{~km} \mathrm{~s}^{-1}$ (W33 B) seem to be outliers.

previous estimated to be spectral type O6 should have a spectral type O7.5. Thus, the star cluster in W33 Main has spectral types ranging from $\mathrm{O} 7.5$ to $\mathrm{B} 1.5$.

Our observations show maser detections in the W33 complex in three different velocity ranges:

- $57-63 \mathrm{~km} \mathrm{~s}^{-1}$ in G012.68-0.18,

- $33-38 \mathrm{~km} \mathrm{~s}^{-1}$ in G012.81-0.19, G012.90-0.24, G012.900.26

- $-5-0 \mathrm{~km} \mathrm{~s}^{-1}$ in G012.81-0.19.

These findings are confirmed by water and hydroxyl maser observations by Genzel \& Downes (1977), Lada et al. (1981), and Argon et al. (2000). Ammonia observations with the Effelsberg $100 \mathrm{~m}$ telescope (Wienen et al. 2012) and from the HOPS survey (Purcell et al. 2012) give line-of-sight velocities of $\sim 55$, $\sim 36, \sim 36$, and $\sim 37 \mathrm{~km} \mathrm{~s}^{-1}$ for G012.68-0.18, G012.81-0.19, G012.90-0.24, and G012.90-0.26, respectively, in accordance with our maser observations. On the other hand, Urquhart et al. (2008) and Chen et al. (2010) detected CO emission peaks at 35,60 , and $52 \mathrm{~km} \mathrm{~s}^{-1}$ in G012.68-0.18, G012.81-0.19, and G012.90-0.26. These results show that both velocity components at $\sim 36$ and $\sim 58 \mathrm{~km} \mathrm{~s}^{-1}$ are spread over the entire complex but the emission at these velocities peaks in different regions. The ammonia observations of Wienen et al. (2012) show the velocity component at $\sim 58 \mathrm{~km} \mathrm{~s}^{-1}$ also at positions in the vicinity of W33 (e.g. G11.94-0.26, G12.74-0.10, G12.90-0.03). This indicates that this velocity component is not unique to the inner W33 complex and probably has a different origin than internal motions (e.g. outflows) in the clouds. The velocity component at $\sim-1 \mathrm{~km} \mathrm{~s}^{-1}$ in G012.81-0.19 was not detected in previous thermal line observations. The arrangement of the water maser spots in this velocity group (see Fig. 6) might suggest that this velocity component is associated with the shock front of an outflow in the active cloud W33 Main (Immer et al., in prep.). 
Although water masers are often found at the base of bipolar outflows, the small radial velocity difference between the masing and the thermal gas in G012.81-0.19, G012.90-0.24, and G012.90-0.26 (see Table 2), the small radial velocity range of the masing gas and the proximity of the masers to submillimeter dust peaks suggests that the 3D motions of the water masers are similar to the motions of the associated central objects and not drastically altered by strong outflows. The only case where a strong outflow could affect the proper motion of the water maser without changing its radial velocity is if the outflow was aligned with the plane of the sky. However, the probability of outflows being in the plane of the sky in all three sources is small. Thus, we assume that the average proper motion of the water maser spots in each source is similar to the motion of its central object. Under this assumption, we can estimate the cloud motions internal to the W33 complex from the motions of the central objects. In G012.68-0.18, we estimated the motion of the central object by fitting a model of expanding outflows to the proper motions of the maser spots. Since the maser spots in the velocity group around $-1 \mathrm{~km} \mathrm{~s}^{-1}$ in G012.81-0.19 have a very different radial velocity than the thermal gas in this cloud, their proper motions probably do not reflect the motion of the central object of the W33 Main cloud. They were thus excluded from the following calculations. We averaged the motions of W33 A (G012.90-0.24, G012.90-0.26), W33 B (G012.68-0.18), and W33 Main (G012.81-0.19-34 $\mathrm{km} \mathrm{s}^{-1}$ ), yielding an estimate of the average proper motion of the com-

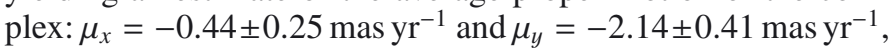
corresponding to $-5 \mathrm{~km} \mathrm{~s}^{-1}$ and $-24 \mathrm{~km} \mathrm{~s}^{-1}$ in the eastward and northward directions, respectively (at the measured distance of $2.40 \mathrm{kpc}$ ). To determine the cloud motions of W33 A and W33 B relative to the W33 Main cloud, the motion of W33 Main was subtracted from the motion of each cloud. Figure 1 shows the cloud motions of W33 A and W33 B in the reference frame of W33 Main on the $870 \mu \mathrm{m}$ dust emission map of the W33 complex.

That W33 A and W33 Main have similar radial velocities suggests that most of the motion of W33 A in the reference frame of W33 Main is in the plane of the sky, tangentially to W33 Main (total speed $\approx 17 \mathrm{~km} \mathrm{~s}^{-1}$ ).

The difference in radial velocity between W33 Main and $\mathrm{W} 33 \mathrm{~B}$ is $\sim 22 \mathrm{~km} \mathrm{~s}^{-1}$. This large velocity component along the line of sight coincidentially is equal to the velocity component in the plane of the sky of $22 \mathrm{~km} \mathrm{~s}^{-1}$ (relative to W33 Main), yielding a total speed of $31 \mathrm{~km} \mathrm{~s}^{-1}$ for W33 B relative to W33 Main. In a forthcoming paper, we will compare the gravitational and kinetic energies of the clouds to determine if W33 A and W33 B are gravitationally bound to W33 Main.

The massive star forming region $\mathrm{G} 012.88+0.48$ appears associated with a molecular cloud at a velocity of $30.9 \mathrm{~km} \mathrm{~s}^{-1}$ (Fig. 11). Thus, both cloud velocity and parallax distance locate this source in the Scutum spiral arm. Since previous studies of G012.88+0.48 assumed a distance of $3.6 \mathrm{kpc}$, its luminosity and mass have been overestimated by a factor of two and should be revised to $L \sim 1.3 \times 10^{5} L_{\odot}$ and $M \sim 1.3 \times 10^{3} M_{\odot}$.

\section{Conclusion}

Trigonometric parallax observations of water masers in the $\mathrm{G} 012.88+0.48$ region and in the massive star forming complex W33 (containing G012.68-0.18, G012.81-0.19, G012.90-0.24, G012.90-0.26) yield distances which are all consistent with $2.40_{-0.15}^{+0.17} \mathrm{kpc}$. A combination of our distance with $\mathrm{CO}$ observations of the Galactic plane by Dame et al. (2001) allows us to locate the W33 complex and G012.88+0.48 in the Scutum spiral arm. We show that W33 is a single star forming complex at about two-thirds the kinematic distance of $3.7 \mathrm{kpc}$. Thus, the luminosity and mass estimates of this region should be lowered to $L \sim 8 \times 10^{5} L_{\odot}$ and $M \sim(0.8-8) \times$ $10^{5} M_{\odot}$. The spectral types in the star cluster in W33 Main should be changed by 1.5 points to later types, yielding spectral types ranging from $\mathrm{O} 7.5$ to $\mathrm{B} 1.5$.

The luminosity and mass values of G012.88+0.48 have also been overestimated in the past and revised values are $L \sim 1.3 \times$ $10^{5} L_{\odot}$ and $M \sim 1.3 \times 10^{3} M_{\odot}$.

Acknowledgements. The authors would like to thank Koichiro Sugiyama for providing the $6.7 \mathrm{GHz}$ methanol maser results of $\mathrm{G} 012.88+0.48$ from the Japanese VLBI Network survey. This work was partially funded by the ERC Advanced Investigator Grant GLOSTAR (247078). This work made use of the Swinburne University of Technology software correlator, developed as part of the Australian Major National Research Facilities Programme and operated under licence (Deller et al. 2007).

\section{References}

Argon, A. L., Reid, M. J., \& Menten, K. M. 2000, ApJS, 129, 159 Beuther, H., Schilke, P., Menten, K. M., et al. 2002, ApJ, 566, 945 Bieging, J. H., Pankonin, V., \& Smith, L. F. 1978, A\&A, 64, 341 Bieging, J. H., Wilson, T. L., \& Downes, D. 1982, A\&AS, 49, 607 Brunthaler, A., Reid, M. J., Menten, K. M., et al. 2011, Astron. Nachr., 332, 461 Burton, W. B., \& Gordon, M. A. 1978, A\&A, 63, 7

Chen, X., Shen, Z.-Q., Li, J.-J., Xu, Y., \& He, J.-H. 2010, ApJ, 710, 150

Dame, T. M., Hartmann, D., \& Thaddeus, P. 2001, ApJ, 547, 792

Davies, B., Lumsden, S. L., Hoare, M. G., Oudmaijer, R. D., \& de Wit, W.-J. 2010, MNRAS, 402, 1504

Deller, A. T., Tingay, S. J., Bailes, M., \& West, C. 2007, PASP, 119, 318

Faúndez, S., Bronfman, L., Garay, G., et al. 2004, A\&A, 426, 97

Fey, A. L., Gordon, D., Jacobs, C. S., et al. 2009, IERS Technical Note, 35, 1

Fujisawa, K., Hachisuka, K., Sugiyama, K., et al. 2012, in IAU Symp. 287, eds. R. S. Booth, E. M. L. Humphreys, \& W. H. T. Vlemmings, 288 Galván-Madrid, R., Zhang, Q., Keto, E., et al. 2010, ApJ, 725, 17

Gardner, F. F., Wilson, T. L., \& Thomasson, P. 1975, Astrophys. Lett., 16, 29

Genzel, R., \& Downes, D. 1977, A\&AS, 30, 145

Goldsmith, P. F., \& Mao, X.-J. 1983, ApJ, 265, 791

Goss, W. M., Matthews, H. E., \& Winnberg, A. 1978, A\&A, 65, 307

Haschick, A. D., \& Ho, P. T. P. 1983, ApJ, 267, 638

Immer, K., Brunthaler, A., Reid, M. J., et al. 2011, ApJS, 194, 25

Jaffe, D. T., Guesten, R., \& Downes, D. 1981, ApJ, 250, 621

Kettenis, M., van Langevelde, H. J., Reynolds, C., \& Cotton, B. 2006, in Astronomical Data Analysis Software and Systems XV, eds. C. Gabriel, C. Arviset, D. Ponz, \& S. Enrique, ASP Conf. Ser., 351, 497

Lada, C. J., Blitz, L., Reid, M. J., \& Moran, J. M. 1981, ApJ, 243, 769

Purcell, C. R., Longmore, S. N., Walsh, A. J., et al. 2012, MNRAS, 426, 1972

Reid, M. J., Menten, K. M., Brunthaler, A., et al. 2009a, ApJ, 693, 397

Reid, M. J., Menten, K. M., Zheng, X. W., et al. 2009b, ApJ, 700, 137

Sato, M., Hirota, T., Reid, M. J., et al. 2010a, PASJ, 62, 287

Sato, M., Reid, M. J., Brunthaler, A., \& Menten, K. M. 2010b, ApJ, 720, 1055

Schuller, F., Menten, K. M., Contreras, Y., et al. 2009, A\&A, 504, 415

Sridharan, T. K., Beuther, H., Schilke, P., Menten, K. M., \& Wyrowski, F. 2002, ApJ, 566, 931

Stier, M. T., Jaffe, D. T., Rengarajan, T. N., et al. 1984, ApJ, 283, 573

Urquhart, J. S., Busfield, A. L., Hoare, M. G., et al. 2008, A\&A, 487, 253

van der Tak, F. F. S., \& Menten, K. M. 2005, A\&A, 437, 947

Wienen, M., Wyrowski, F., Schuller, F, et al. 2012, A\&A, 544, A146

Xu, Y., Reid, M. J., Zheng, X. W., \& Menten, K. M. 2006, Science, 311, 54

Xu, Y., Moscadelli, L., Reid, M. J., et al. 2011, ApJ, 733, 25 